\title{
Tuberculosis in Well-Nourished Children in Calabar, Nigeria
}

\author{
Atana Ewa1, Aniefon Umana², Nta Okoi ${ }^{3}$, Francis Akpan', Carlistus Enyuma1 \\ ${ }^{1}$ Department of Paediatrics, University of Calabar/Teaching Hospital, Calabar, Nigeria \\ ${ }^{2}$ Department of Otorhinolaryngology, University of Calabar/Teaching Hospital, Calabar, Nigeria \\ ${ }^{3}$ Department of Family Medicine, University of Calabar/Teaching Hospital, Calabar, Nigeria \\ Email: atanaewa@yahoo.com
}

Received 19 March 2015; accepted 26 June 2015; published 30 June 2015

Copyright (C) 2015 by authors and Scientific Research Publishing Inc.

This work is licensed under the Creative Commons Attribution International License (CC BY). http://creativecommons.org/licenses/by/4.0/

(c) (i) Open Access

\begin{abstract}
Tuberculosis has traditionally been found to occur with weight loss or at least failure to gain weight. Atypical and unusual presentations of various types of tuberculosis have been reported but limited data is available on tuberculosis in well-nourished children with no weight loss. Two case reports from our experience highlight well-nourished children with histologically diagnosed tuberculosis that presented with atypical symptoms and no evidences of weight loss. The usefulness of histological diagnosis cannot be overemphasized. This report aims to highlight this unusual presentation of tuberculosis so as to increase the index of suspicion for early diagnosis and hence, prompt treatment of tuberculosis in this group of children.
\end{abstract}

\section{Keywords}

Tuberculosis, Weight Loss, Atypical, Well-Nourished, Histological Diagnosis

\section{Introduction}

Tuberculosis (Tb) is the leading cause of death in both adults and children worldwide [1]. It has been a global emergency, especially in sub-Saharan Africa [2]. Published reports are common in adults but there are no recent epidemiological data on global tuberculosis burden in children. Adults with tuberculosis are often easily recognized due to typical symptoms but children often pose diagnostic difficulties due to atypical features [1] [3]. Also adolescents may have peculiar TB clinical manifestations and in some cases, they may be asymptomatic even in confirmed tuberculosis [2]. The purpose of this report is to highlight the unusual presentation of tuberculosis in some children so as to increase the index of suspicion for early diagnosis of tuberculosis in this group of children. 


\section{Case Reports}

Case 1: An 8-year-old boy presented with difficulty in breathing through the nose for 1 year, gradually getting worse and recently associated with snoring, mouth breathing, restlessness and apneic attacks during sleep. He was also noticed sometimes to distend/extend the neck while sleeping in order to breathe well. He is the 2nd of 3 children and has been apparently well before these complaints. He had received BCG at birth.

His first presentation was about a year ago with cough, chest pain and fever. He was well nourished, afebrile and not in respiratory distress with mild tenderness over the right mid lung zone. Only vesicular breath sounds were heard. Other systems were normal. A diagnosis of probable pneumonia was made. Chest X-ray showed patchy and hilar opacities in both lungs. Full blood count was normal but Erythrocyte Sedimentation Rate (ESR) was $103 \mathrm{~mm} / \mathrm{hr}$. He was made to complete amoxicillin/clavulanate, which he had earlier started, and later azithromycin after which the symptoms subsided. A repeat ESR had dropped to $60 \mathrm{~mm} / \mathrm{hr}$ and CXR had hilar opacities, which incidentally had persisted through three previous X-rays. The diagnosis of pulmonary tuberculosis was considered at that time but couldn't be substantiated nor confirmed due to lack of signs and symptoms as well as clinical improvement with sustained weight gain.

Physical examination at this presentation revealed a well-nourished boy with noisy breathing, cervical lymphadenopathy with enlarged kissing tonsils, which were not inflamed. His weight was $36 \mathrm{~kg}$ (just below 97th percentile for age). The diagnosis of Obstructive Sleep Apnea secondary to Obstructive adenoids and tonsils was made. He was referred to the Otorhinolaryngologist who after various investigations performed an adenotonsillectomy. The findings were very large obstructive adenoids, massively enlarged tonsils and healthy mucosa. The tissue histology report revealed caseous necrosis and numerous granulomas consisting of lymphocytes, fibroblasts, plasma cells, Langerhans and foreign body giant cells. The diagnosis was tuberculosis of the larynx with probable PTB because of the persistent radiological findings. He was treated with anti-tuberculosis short course chemotherapy comprising Rifampicin, Isoniazid, Pyrazinamide and Ethambutol for the first 2 months followed by Rifampicin and Isoniazid for the remaining 4 months. The cervical lymphadenitis and radiological findings all returned to normal and he was declared cured. The final diagnosis was Pulmonary Tuberculosis with $\mathrm{Tb}$ of the larynx (disseminated tuberculosis).

Case 2: A 14-year-old female student presented with cervical swelling of 4 months duration that was slow growing and painless without cough or fever until one month before presentation, when cough was noticed. There was neither associated weight loss nor contact with a person with tuberculosis. No other swelling was seen anywhere else in the body. She had received all childhood vaccines but no BCG scar was evident. She is the 3rd of 4 children with excellent academic performance in school.

Physical examination showed a well-nourished girl weighing $57 \mathrm{~kg}$ (above 50th percentile for age) with multiple right-sided cervical lymphadenopathies involving the supraclavicular and posterior triangle of the neck. The largest node measured $8 \mathrm{~cm}$ by $6 \mathrm{~cm}$, firm, and nontender, matted together and not attached to the overlying skin. An initial diagnosis was Hodgkin's lymphoma to rule out tuberculosis.

The chest X-Ray had right hilar opacity persisting in two serial X-rays. A Mantoux test measured $60 \mathrm{~mm}$ with ulceration. Full blood count was normal with a packed cell volume of $35.5 \%$ and Erythrocyte Sedimentation Rate of $96 \mathrm{~mm} / \mathrm{hr}$. HIV screening was non-reactive. An excision biopsy of the lymph node showed a caseating tumour measuring $4 \times 2 \mathrm{~cm}$ from the posterior triangle of the neck. Histology report revealed caseous necrosis, numerous granuloma, foreign body and tissue Langerhan's giant cells, residual lymphoid tissue and no malignant cells. The final diagnosis was tuberculous adenitis with probable Pulmonary Tuberculosis. She was treated with short course anti-tuberculosis chemotherapy comprising of Rifampicin, Isoniazid, Pyrazinamide and Ethambutol for the first 2 months followed by Rifampicin and Isoniazid for the remaining 4 months. Follow-up visits during and after treatment showed complete resolution of the cervical lymphadenitis. She was declared cured and discharged from the respiratory clinic, to be seen in the Paediatric clinics when necessary.

\section{Discussion}

Childhood tuberculosis contributes only 3\% - 6\% of all the burden of illness caused by tuberculosis in developed countries, but in developing countries the figures are much higher (15\% - 20\%) [1]. Despite this, it remains a neglected research area [1] with very few studies investigating and studying these children [2] [3]. Published reports are common in adults but there are no recent epidemiological data on global tuberculosis burden in children. Adult tuberculosis cases are often easily recognized due to typical symptoms but children with tuber- 
culosis often pose diagnostic difficulties due to atypical features [1] [3]. The first case (Case 1) had a delayed diagnosis because he was well-nourished despite having cough, chest pain and fever with hilar and perihilar opacities on chest X-ray as far back as one year before he was diagnosed. This radiological finding persisted through three serial X-rays over the period of one year. Also, the clinical response following the use of azithromycin and the improvement in Erythrocyte Sedimentation Rate contributed to the delay in diagnosing tuberculosis. At this point, he could have had a latent tuberculosis infection. There are reports that most Tb cases in children present as latent tuberculosis infection (LTBI) and it is known that infected children are reservoirs from which many adult tuberculosis cases will arise [1]. Also the incidence and prevalence of Tb in children are underestimated due to difficulties in collecting samples and lack of efficient method to detect the disease [1]. For example, there are no invasive tests to confirm a diagnosis of laryngeal tuberculosis. Biopsy of the primary growth itself is diagnostic and may show caseating granulomatous inflammation. Microbiological confirmation may not always be possible [4]. This also emphasizes the usefulness of tissue histology after surgical removal. Case 1 had no cough in this presentation and so sputum collection was not feasible. The only available specimens were the tonsils and adenoids that were removed and sent for histology. He would have remained undiagnosed if these were not sent for histology. The second case (Case 2) in particular did not have fever and cough and was only present three months after the neck swelling. Little wonder about the working diagnosis of Hodgkin's Lymphoma. Her Mantoux response was alarming and lymph node histology supported the diagnosis of tuberculosis.

A recent report described tuberculosis in well nourished children with no weight loss, anorexia nor contact with cases of tuberculosis [5]. Another report also described tuberculous osteomyelitis with no weight loss, nor pulmonary symptoms and normal chest radiograph [6].

Traditionally, tuberculosis is a chronic disease with various clinical manifestations [3]. Weight loss or at least failure to gain weight is a constant feature [7]. Cough, fever, dyspnea, chest pain, loss of appetite, weakness, night sweats and malaise are also common [3]. Uncommon and very atypical is the absence of weight loss as seen in these two reported cases.

\section{Conclusion}

Tuberculosis is a disease known to manifest with weight loss or at least failure to gain weight. The two case reports from our experience highlight well-nourished children with histologically diagnosed tuberculosis who presented with atypical symptoms and no evidences of weight loss. One of them actually gained weight before commencing treatment for tuberculosis. A high index of suspicion is therefore needed for early diagnosis and prompt treatment of tuberculosis in such cases.

\section{Conflict of Interest}

None

\section{References}

[1] López Ávalos, G.G. and Montes de Oca, E.P. (2012) Classic and New Diagnostic Approaches to Childhood Tuberculosis. Journal of Tropical Medicine, 2012, Article ID: 818219. http://dx.doi.org/10.1155/2012/818219

[2] Waako, J., Verver, S., Wajja, A., Ssengooba, W., Joloba, M.L., Colebunders, R., et al. (2013) Burden of Tuberculosis Disease among Adolescents in a Rural Cohort in Eastern Uganda. BMC Infectious Diseases, 13, 349. http://dx.doi.org/10.1186/1471-2334-13-349

[3] Aharmin, M., Bouti, K., Soualihi, M., Hanim, F.Z., Mara, K., Zahroni, R., et al. (2014) Pediatric Tuberculosis at Moulay Youssef University Hospital, Morrocco. International Journal of Medicine and Surgery, 1, 17-22.

[4] Verma, S.K., Verma, S.K. and Sanjay. (2007) Laryngeal Tuberculosis Clinically Similar to Laryngeal Cancer. Lung India, 24, 87-89.

[5] Senanayake, M.P. and Karunaratne, I. (2014) A Child Presenting with Tuberculous Spondylitis in a Single Third Cervical Vertebrae: A Case Report. Journal of Medical Case Reports, 8, 284. http://dx.doi.org/10.1186/1752-1947-8-284

[6] Khan, G.M.K., Humail, S.M. and Hafeez, K. (2014) Primary Diaphyseal Tuberculous Osteomyelitis of the Tibia. Professional Medical Journal, 21, 1282-1284.

[7] Ray, S., Talukdar, A., Kimdu, S., Kharma, D. and Sonthalia, N. (2013) Diagnosis and Management of Miliary Tuberculosis: Current State and Future Perspectives. Therapeutics and clinical Risk Management, 2013, 9-26.

http://dx.doi.org/10.2147/TCRM.S29179 